\title{
Effects of Radiation and Variable Fluid Viscosity on Flow and Heat Transfer along a Symmetric Wedge
}

\author{
S. Mukhopadhyay \\ Department of Mathematics, M.U.C. Women's College, Burdwan-713104, W. B., India. \\ Email:swati_bumath@yahoo.co.in
}

(Received April 22, 2008; accepted January 9, 2009)

\begin{abstract}
Radiation effects on boundary layer flow and heat transfer of a fluid with variable viscosity along a symmetric wedge is presented here. Fluid viscosity is assumed to vary as a linear function of temperature. The symmetry groups admitted by the corresponding boundary value problem are obtained by using a special form of Lie group transformations viz. scaling group of transformations. A third order and a second order coupled ordinary differential equation system corresponding to the momentum and the energy equations are obtained. These equations are then solved numerically. With the increase of temperature-dependent fluid viscosity parameter (i.e. with decreasing viscosity), the fluid velocity increases up to the cross-over point $\left(\eta=\eta_{0}\right)\left(\eta_{0} \approx 0.90\right.$ is the nearest numerical value of the cross-over point) and after the crossing over point the fluid velocity is found to decrease but the temperature increases at a particular point. The significant finding of this study is that, due to variable fluid viscosity, flow separation is controlled. The temperature decreases with increasing value of radiation parameter and Prandtl number.
\end{abstract}

Keywords: Scaling group of transformations, wedge flow, radiation effect, temperature dependent fluid viscosity.

\section{NOMENCLATURE}

$\begin{array}{ll}A & \text { fluid viscosity variation parameter } \\ c_{p} & \text { specific heat } \\ F & \text { non dimensional stream function } \\ k^{*} & \text { absorption coefficient } \\ m & \text { Falkner-Skan exponent } \\ \operatorname{Pr} & \text { Prandtl number } \\ Q & \text { radiative heating parameter } \\ q_{r} & \quad \text { radiative heat flux } \\ T, T_{w}, T_{\infty} \text { temperature of the fluid, wall, free stream }\end{array}$

\section{INTRODUCTION}

The study of hydrodynamic flow and heat transfer along a symmetric wedge has gained considerable attention due to its vast applications in industry and its important bearings on several technological and natural processes. The momentum boundary layer equation for Falkner-Skan flow past a wedge with potential flow velocity was first studied by Falkner and Skan (1930). Hartree (1937) later investigated the same problem and found the similarity solutions. Koh and Hartnett (1959) have obtained the skinfriction and heat transfer for flow over a porous wedge with suction subjected to variable wall temperature. There is a large number of investigations on free, forced and mixed

\author{
Greek symbols \\ $\alpha, \gamma \quad$ transformation parameters \\ $\eta \quad$ similarity variable \\ $\kappa \quad$ thermal conductivity \\ $\mu, \mu^{*}$ dynamic, reference viscosity \\ $v \quad$ reference kinematic viscosity \\ $\psi \quad$ stream function \\ $\sigma \quad$ Stefan-Boltzman constant \\ $\rho \quad$ density of the fluid \\ $\theta \quad$ non dimensional temperature
}

convective flow over a wedge (Smith 1967, Williams and Rhyne 1980, Hossain et al. 2006).

It is well known that the occurrence of flow separation has several undesirable effects in so far as it leads to an increase in the drag on a body immersed in the flow and adversely affects the heat transfer from the surface of the body. Several methods have been developed for the purpose of artificial control of flow separation.

Radiative effects have important applications in physics and engineering. The radiation heat transfer effects on different flows are very important in space technology and high temperature processes. But very little is known about 
the effects of radiation on the boundary layer. Thermal radiation effects may play an important role in controlling heat transfer in polymer processing industry where the quality of the final product depends on the heat controlling factors to some extent. High temperature plasmas, cooling of nuclear reactors, liquid metal fluids, power generation systems are some important applications of radiative heat transfer from a wall to conductive gray fluids. The effect of radiation on heat transfer problems have been studied by Hossain and Takhar (1996), Takhar et al. (1996).

In all of the above mentioned studies, fluid viscosity was assumed to be constant. However, it is known that the physical properties of the fluid may change significantly with temperature. For lubricating fluids, heat generated by the internal friction and the corresponding rise in temperature affects the viscosity of the fluid and so the fluid viscosity can no longer be assumed constant. The increase of temperature leads to a local increase in the transport phenomena by reducing the viscosity across the momentum boundary layer and so the heat transfer rate at the wall is also affected. When the fluid is assumed to have constant properties, the problem reduces to uncoupled laminar boundary layer-flow and the fluid velocity field is unaffected by the temperature changes but the problem becomes coupled when the thermo-physical properties depend on temperature. Such a flow has direct relevance in aerodynamics (Hossain et al. 2006). Therefore, to predict the flow behaviour accurately it is necessary to take into account the viscosity variation for incompressible fluids.

Hossain et al. (2000) considered the flow of viscous incompressible fluid with temperature dependent viscosity and thermal conductivity past a permeable wedge with uniform surface heat flux. Of late, Pantokratoras (2006) studied the Falkner-Skan flow problem with variable fluid viscosity. A new dimension is added to the above mentioned problem by considering the thermal radiation.

The present work deals with the flow of viscous incompressible fluid having variable viscosity and heat transfer along a symmetric wedge. Using similarity variable and similarity solution solutions, a third order and a second order coupled ordinary differential equation system corresponding to the momentum and the energy equations are derived. These equations are solved numerically using a shooting method.

The effects of the temperature-dependent fluid viscosity parameter, radiation parameter, the influence of Prandtl number on temperature fields of the fluid are investigated and analysed with the help of their graphical representations.

\section{EQUATIONS OF Motion}

We consider a steady, two-dimensional, laminar boundarylayer flow of viscous incompressible fluid past a symmetrical sharp wedge with velocity given by $\bar{u}_{e}(\bar{x})=U_{\infty}\left(\frac{\bar{x}}{L}\right)^{m} \quad$ for $\quad m \leq 1 \quad$ where $L \quad$ is the characteristic length and $m$ is the velocity exponent related to the included angle $\pi \beta$ by $m=\frac{\beta}{2-\beta}$. For $m<0$, the solution becomes singular at $\bar{x}=0$, while for $m \geq 0$, the solution can be defined for all values of $\bar{x}$. The governing equations of such type of flow are, in the usual notations,

$\frac{\partial \bar{u}}{\partial \bar{x}}+\frac{\partial \bar{v}}{\partial \bar{y}}=0$

$\bar{u} \frac{\partial \bar{u}}{\partial \bar{x}}+\bar{v} \frac{\partial \bar{u}}{\partial \bar{y}}=\bar{u}_{e} \frac{\partial \bar{u}_{e}}{\partial \bar{x}}+\frac{1}{\rho} \frac{\partial \mu}{\partial T} \frac{\partial T}{\partial \bar{y}} \frac{\partial \bar{u}}{\partial \bar{y}}+\frac{\mu}{\rho} \frac{\partial^{2} \bar{u}}{\partial \bar{y}^{2}}$,

$\bar{u} \frac{\partial T}{\partial \bar{x}}+\bar{v} \frac{\partial T}{\partial \bar{y}}=\frac{\kappa}{\rho c_{p}} \frac{\partial^{2} T}{\partial \bar{y}^{2}}-\frac{1}{\rho c_{p}} \frac{\partial q_{r}}{\partial \bar{y}}$,

when the viscous dissipation term in the energy equation is neglected. Here $\bar{u}$ and $\bar{v}$ are the components of velocity respectively in the $\bar{x}$ and $\bar{y}$ directions. Using the Rosseland approximation for radiation (Brewster, 1972) we can write $q_{r}=-\frac{4 \sigma}{3 k^{*}} \frac{\partial T^{4}}{\partial \bar{y}}$. Assuming the temperature difference within the flow is such that $T^{4}$ may be expanded in a Taylor series about $T_{\infty}$ and neglecting higher orders we get $T^{4} \equiv 4 T_{\infty}^{3} T-3 T_{\infty}^{4}$. Therefore, the Eq. (3) becomes

$\bar{u} \frac{\partial T}{\partial \bar{x}}+\bar{v} \frac{\partial T}{\partial \bar{y}}=\frac{\kappa}{\rho c_{p}} \frac{\partial^{2} T}{\partial \bar{y}^{2}}+\frac{16 \sigma T_{\infty}^{3}}{3 \rho c_{p} k^{*}} \frac{\partial^{2} T}{\partial \bar{y}^{2}}$.

The appropriate boundary conditions for the problem are given by

$u=0, v=0, T=T_{w}$ at $y=0$,

$\bar{u} \rightarrow \bar{u}_{e}(\bar{x}), T \rightarrow T_{\infty}$ as $\bar{y} \rightarrow \infty$.

\section{Method OF Solution}

JIntroducing

$$
\begin{aligned}
& x=\frac{\bar{x}}{L}, y=\operatorname{Re}_{L}^{1 / 2} \frac{\bar{y}}{L}, u=\frac{\bar{u}}{U_{\infty}}, \\
& v=\operatorname{Re}_{L}^{1 / 2} \frac{\bar{v}}{U_{\infty}}, u_{e}=\frac{\bar{u}_{e}}{U_{\infty}}, \operatorname{Re}_{L}=\frac{U_{\infty} L}{v}
\end{aligned}
$$

In the above Eqs. (1), (2) and (4) we get

$$
\begin{aligned}
& \frac{\partial u}{\partial x}+\frac{\partial v}{\partial y}=0 \\
& u \frac{\partial u}{\partial x}+v \frac{\partial u}{\partial y}=u_{e} \frac{\partial u_{e}}{\partial x}+\frac{1}{\mu^{*}} \frac{\partial \mu}{\partial T} \frac{\partial T}{\partial y} \frac{\partial u}{\partial y}+\frac{\mu}{\mu^{*}} \frac{\partial^{2} u}{\partial y^{2}} \\
& u \frac{\partial T}{\partial x}+v \frac{\partial T}{\partial y}=\frac{\kappa}{v \rho c_{p}} \frac{\partial^{2} T}{\partial y^{2}}+\frac{16 \sigma T_{\infty}^{3}}{3 v \rho c_{p} k^{*}} \frac{\partial^{2} T}{\partial y^{2}}
\end{aligned}
$$

where $v=\mu^{*} / \rho \mu^{*}$ is the constant value of the coefficient of viscosity far away from the surface. The boundary conditions Eqs. (5a) and (5b) now become

$$
\begin{aligned}
& u=0, v=0, T=T_{w} \text { at } y=0, \\
& u \rightarrow u_{e}(x), T \rightarrow T_{\infty} \text { as } y \rightarrow \infty .
\end{aligned}
$$

The velocity of the fluid over the wedge is now given by $u_{e}(x)=x^{m}$, for $m \leq 1$. 
We now introduce the following relations for $u, v$ and $\theta$ as

$$
u=\frac{\partial \psi}{\partial y}, v=-\frac{\partial \psi}{\partial x} \text { and } \theta=\frac{T-T_{\infty}}{T_{w}-T_{\infty}} .
$$

I use the temperature dependent fluid viscosity given by (Mukhopadhyay et al. 2005),

$$
\mu=\mu^{*}\left[a+b\left(T_{w}-T\right)\right]
$$

where $a$,b are constants and $\mathrm{b}>0$.

Using the Eqs. (11) and (12) in the boundary layer Eq. (8) and in the energy Eq. (9) we get the following equations

$$
\begin{aligned}
& \frac{\partial \psi}{\partial y} \frac{\partial^{2} \psi}{\partial x \partial y}-\frac{\partial \psi}{\partial x} \frac{\partial^{2} \psi}{\partial y^{2}}=u_{e} \frac{\partial u_{e}}{\partial x}-A \frac{\partial \theta}{\partial y} \frac{\partial^{2} \psi}{\partial y^{2}} \\
& +[a+A(1-\theta)] \frac{\partial^{3} \psi}{\partial y^{3}},
\end{aligned}
$$

and

$$
\frac{\partial \psi}{\partial y} \frac{\partial \theta}{\partial x}-\frac{\partial \psi}{\partial x} \frac{\partial \theta}{\partial y}=\left(\frac{\kappa}{v \rho c_{p}}+\frac{16 \sigma T_{\infty}^{3}}{3 v \rho c_{p} k^{*}}\right) \frac{\partial^{2} \theta}{\partial y^{2}}
$$

where $A=b\left(T_{w}-T_{\infty}\right)$.

The boundary conditions Eqs. (10a) and (10b) then become

$\frac{\partial \psi}{\partial y}=0, \frac{\partial \psi}{\partial x}=0, \theta=1$ at $y=0$.

$\frac{\partial \psi}{\partial y} \rightarrow u_{e}(x)=x^{m}, \theta \rightarrow 0$ as $y \rightarrow \infty$.

Introduce the following relations

$$
\psi(x, y)=x^{\alpha} F(\eta), \theta(x, y)=G(\eta), \quad \eta=\frac{y}{x^{\gamma}}
$$

in momentum and energy equations. Then the momentum and energy equations give $\alpha=1-\gamma$ and the momentum equation also gives $\alpha-3 \gamma=2 m-1$, the solution of which is $\alpha=\frac{1+m}{2}, \quad \gamma=\frac{1-m}{2}$ and the resulting governing equations then become

$$
\begin{aligned}
& m F^{\prime 2}-\frac{m+1}{2} F F^{\prime \prime}=m-A G^{\prime} F^{\prime \prime} \\
& +(a+A) F^{\prime \prime \prime}-A G F^{\prime \prime \prime} \\
& (3+4 Q) G^{\prime \prime}+3\left(\frac{m+1}{2}\right) \operatorname{Pr} F G^{\prime}=0
\end{aligned}
$$

where $\operatorname{Pr}=\mu^{*} c_{p} / \kappa$ is the Prandtl number, $Q=4 \sigma T_{\infty}^{3} / \kappa k^{*}$ is the radiative heating parameter.

The boundary conditions take the following form

$$
\begin{aligned}
& F^{\prime}=0, F=0, G=1 \text { at } \eta=0 \\
& \text { and } F^{\prime} \rightarrow 1, G \rightarrow 0 \text { as } \eta \rightarrow \infty .
\end{aligned}
$$

The above Eqs. (17) and (18) along with boundary conditions are solved numerically by shooting method.

\section{Results AND Discussions}

In order to analyse the results, numerical computation has been carried out for various values of the temperaturedependent viscosity parameter (A), Falkner-Skan exponent $(m)$, radiative heating parameter $(Q)$ and the Prandtl number Pr.

For illustrations of the results, numerical values are plotted in the Fig. 1 to Fig. 6 . In all cases we take $\mathrm{a}=1$.

To test the accuracy of the present method, results are compared with those available in the literature. We see that as viscosity becomes constant inside the boundary layer, our profiles tend to coincide with the similarity profiles shown in the literature.

In order to assess the accuracy of the method, the results (in the absence of radiation) are also compared with those of Pantokratoras (2006). Our results with $A=0$ (in the absence of thermal radiation) are found to agree well with those of Pantokratoras (2006) when the viscosity tends to become constant inside the boundary layer. For the sake of brevity, the comparison in detail is given in Table 1.

Now, the result for the variation of the temperaturedependent fluid viscosity parameter $\mathrm{A}$ is presented. In Fig. 1, velocity profiles are shown for different values of A $(\mathrm{A}=0,5,10,20)$ with $m=0.5, Q=2$ and $\operatorname{Pr}=0.5$. The velocity curves show that the rate of momentum transport increases with the increase in $\mathrm{A}$.

It clearly indicates that with the increasing value of A i.e. with the decreasing viscosity, the fluid velocity increases up to the cross-over point ( $\left.\eta=\eta_{0} \approx 0.90\right)$ and after this point the velocity is found to decrease with increasing $A$ at a particular value of $\eta$. This can be explained physically as the parameter A increases, the fluid viscosity decreases resulting the increment of the boundary layer thickness.

But the temperature is found to increase for any non-zero fixed value of $\eta$ with the increase of $\mathrm{A}(\mathrm{A}=0,5,10,20)$ when $m=0.5, Q=2$ and $\operatorname{Pr}=0.5$ (Fig. 2). It is very clear from the figure that the temperature decreases with the increasing value of $\eta$ for all values of A considered here.

Decrease in $G(\eta)$ means a decrease in the velocity of the fluid particles. So in this case the fluid particles encounter two different phenomena: (i) fluid velocity increases due to decrease in the fluid viscosity (with increasing A) and (ii) the fluid velocity decreases due to decrease in temperature $G(\eta)$. Near the wall, as the temperature $G$ is high, the first cause dominates and far away from the wall, where $G$ is low, the second cause is dominant.

Now we concentrate on the velocity and temperature distribution for the variation of Falkner-Skan exponent $m$ in the absence and presence of temperature-dependent fluid viscosity parameter $\mathrm{A}$.

It is worth mentioning that $\beta$ is a measure of the pressure gradient. If $\beta$ is positive, the pressure gradient is negative 
or favorable, and negative $\beta$ denotes a positive pressure gradient (adverse).

Fig. 3a presents the effects of increasing $m$ ( $m=-0.091$, $0.06,0,0.1,0.3,0.5,0.9$ ) on the fluid velocity when the fluid viscosity is uniform i.e. $A=0$. With the increasing value of the exponent $m$ ( $\mathrm{A}=0, Q=0, \operatorname{Pr}=0.5$ ), the velocity is found to increase. It is noted that the boundary layer thickness decreases as $m$ increases, hence it gives rise to the velocity gradient, which in turn causes to increase the skin friction. As $m$ decreases from zero, the positive value of $\partial^{2} u / \partial y^{2}$ at the wall increases and this, together with the constraint $u \rightarrow U$ as $y \rightarrow \infty$, leads to a continual decrease of $(\partial u / \partial y)_{y=0}$. At $m=-0.091$ (this is the smallest value of $m$ for which a complete numerical solution exists in this case) the velocity gradient at the wall vanishes i.e. the frictional force at the wall is zero. This is the criterion of the occurrence of flow separation. It is found that in case of accelerated flow ( $m>0$ ), the velocity profiles have no point of inflexion whereas in case of decelerated flow $(m<0)$, they exhibit a point of inflexion. Flow separation occurs at $m=-0.091$ (Fig. 3a).

It is observed that for $m=-0.0904$ an isothermal FalknerSkan solution first encounters separation whereas it separates for $m=-0.091$ when $Q=0$.

In presence of temperature dependent fluid viscosity (A=10), the effects of increasing $m(m=-0.091,-0.06,0$, $0.3,0.5,0.9$ ) are exhibited in Fig. 3b. In this case the velocity profiles have no point of inflexion in case of accelerated as well as decelerated flows. So a significant result is obtained that in presence of temperature dependent fluid viscosity, flow separation can be prevented.

It is noticed that the temperature $G(\eta)$ in boundary layer decreases with the increasing values of $m$ (in case of both uniform and non-uniform fluid viscosity) since the thermal boundary layer thickness increases with $m$ which causes a decrease in the rate of heat transfer.

Figure 4 exhibits that flow separation can be controlled in presence of thermal radiation. This is in agreement with the result of Grosan and Pop (2007).

Figure 5 demonstrates the effects of radiation parameter $Q$ on temperature field in presence of variable fluid viscosity $(\mathrm{A}=10)$.

The temperature $G(\eta)$ increases as thermal radiation $Q(Q=0.2,0.3,0.5,1)$ increases (Fig. 5). This is in agreement with the physical fact that the thermal boundary layer thickness decreases with increasing $Q$. The effect of radiation in the thermal boundary layer Eq. (9) is equivalent to an increased thermal diffusivity, i.e. $\frac{\operatorname{Pr}}{\left(1+\frac{4}{3} Q\right)}$ in Eq. (18) can be considered as an effective Prandtl number which reduces with increasing values of $Q$. The local Nusselt number for heat transfer in the present case is defined by

$$
N u_{x}=-x\left(\frac{\partial T}{\partial y}\right)_{y=0} /\left(T_{w}-T_{\infty}\right) .
$$

Figure 6 shows the variation of rate of heat transfer with the viscosity variation parameter $\mathrm{A}$ (for $\operatorname{Pr}=0.5$ ). It is very clear that the rate of heat transfer decreases with viscosity variation parameter A, radiation parameter $Q$ (for $m=0.5$ ). It also decreases with increasing values of $m$ (for $Q=2$ ). The thermal boundary layer thickness increases with parameters related to viscosity variation \& thermal radiation and also with the exponent $m$ and reduces the heat transfer coefficient.

\section{CONCLUSION}

The present study gives the similarity solutions for steady boundary layer flow and radiative heat transfer over a symmetric wedge in presence of temperature-dependent fluid viscosity. The effect of increasing temperaturedependent fluid viscosity parameter on a viscous incompressible fluid is to increase the flow velocity up to the cross-over point $\eta_{0}$. The results pertaining to the present study indicate that due to radiation, the rate of heat transfer decreases. It is noticed that the fluid velocity increases with increasing $m$ and flow separation can be controlled in presence of variable fluid viscosity. The temperature in the boundary layer decreases with increasing $m$.

\section{ACKNOWLEDGMENT}

The author gratefully acknowledges the financial support of University Grants Commission (UGC), Eastern Regional Office, Kolkata, W.B., India (Project No. F. PSW-104 /06-07) for pursuing this work. The author is thankful to the honorable reviewers for their constructive suggestions for improvement of the paper.

\section{REFERENCES}

Brewster, M. Q. (1972). Thermal radiative transfer properties. John Wiley and Sons.

Falkner, V.M. and S.W. Skan (1930). Some approximate solutions of the boundary-layer equations, Philosophical Magazine ARCRM 1314, 12, 865-896.

Grosan, T. and I. Pop (2007). Thermal radiation effect $n$ fully developed mixed convection flow in a vertical channel. Technische Mechanik 27 (1), 37-47.

Hartree, D. R. (1937). On equations occurring in Falkner and Skan's approximate treatment of the equations of boundary layer. Proceedings of Cambridge Philosophical Society 33, 223-239.

Hossain, M.A., S. Bhowmik, and R.S.R. Gorla (2006). Unsteady mixed - convection boundary layer flow along a symmetric wedge with variable surface temperature. International Journal of Engineering Science 44, 607-620.

Hossain, M.A., M.S. Munir, D.A.S. Rees (2000). Flow of viscous incompressible fluid with temperature 
S. Mukhopadhyay / JAFM, Vol. 2, No. 2, pp. 29-34, 2009.

dependent viscosity and thermal conductivity past a permeable wedge with uniform surface heat flux. International Journal of Thermal Sciences 39, 635644.

Hossain, M.A. and H.S. Takhar (1996). Radiation effect on mixed convection along a vertical plate with uniform surface temperature. International Journal of Heat and Mass Transfer 31, 243-248.

Koh, J.C.Y. and J.P. Hartnett (1959). Skin-friction and heat transfer for incompressible laminar flow over porous wedges with suction and variable wall temperature. International Journal of Heat Mass Transfer 2, 185198.

Mukhopadhyay, S., G.C. Layek, and S.A. Samad (2005). Study of MHD boundary layer flow over a heated stretching sheet with variable viscosity. International Journal of Heat Mass Transfer 48, 4460-4466.

Pantokratoras, A. (2006). The Falkner-Skan flow with constant wall temperature and variable viscosity. International Journal of Thermal Sciences 45(4), 378389.

Smith, S.H. (1967). The impulsive motion of a edge in a viscous fluid. Journal of Applied Mathematics Physics (ZAMP) 18, 508-522.

Takhar, H.S., R.S.R. Gorla and V.M. Soundalgekar (1996). Radiation effects on MHD free convection flow of a gas past a semi-infinite vertical plate. International Journal of Numerical Methods of Heat and Fluid Flow 6(2),77-83.

Williams, J.C. and T.B. Rhyne (1980). Boundary-layer development on a wedge impulsively set into motion. SIAM Journal of Applied Mathematics 38, 215-224.

Table 1 Values of $f^{\prime \prime}(0)$ and $\theta^{\prime}(0)$ for $\mathrm{A}=0, \mathrm{Q}=0$ and $\mathrm{Pr}=1$

\begin{tabular}{|c|c|c|c|c|c|c|}
\hline \multirow{2}{*}{} & \multicolumn{2}{|c|}{$m=-0.05$} & \multicolumn{2}{c|}{$m=0$} & \multicolumn{2}{c|}{$m=1 / 3$} \\
\cline { 2 - 7 } & $f^{\prime \prime}(0)$ & $\theta^{\prime}(0)$ & $f^{\prime \prime}(0)$ & $\theta^{\prime}(0)$ & $f^{\prime \prime}(0)$ & $\theta^{\prime}(0)$ \\
\hline Present study & 0.2135 & 0.2994 & 0.3320 & 0.3320 & 0.7574 & 0.4401 \\
\hline $\begin{array}{c}\text { Pantokratoras (2006) } \\
\text { (with constant viscosity) }\end{array}$ & 0.2135 & 0.2994 & 0.3320 & 0.3320 & 0.7574 & 0.4401 \\
\hline
\end{tabular}


S. Mukhopadhyay / JAFM, Vol. 2, No. 2, pp. 29-34, 2009.

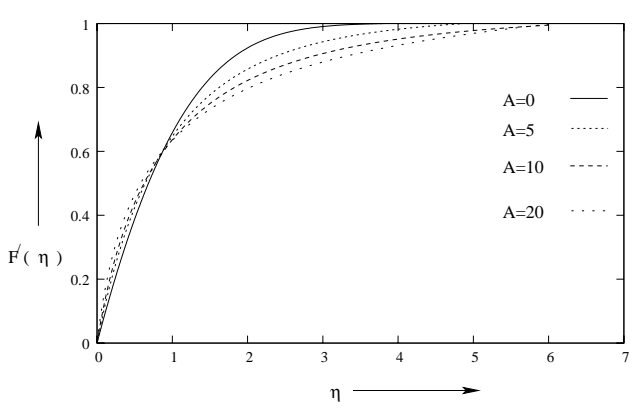

Fig. 1: Variation of velocity $\mathrm{F}^{\prime}(\eta)$ with $\eta$ for several values of A with $\mathrm{a}=1, \mathrm{~m}=0.5, \mathrm{Q}=2$ and $\mathrm{Pr}=0.5$.

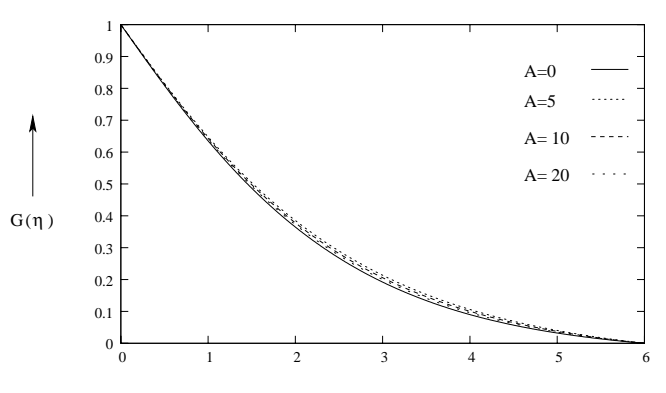

Fig. 2: Distribution of temperature $G(\eta)$ against $\quad$ for several values of $\mathrm{A}$ with $\mathrm{m}=0.5, \mathrm{Q}=2$ and $\mathrm{Pr}=0.5$.

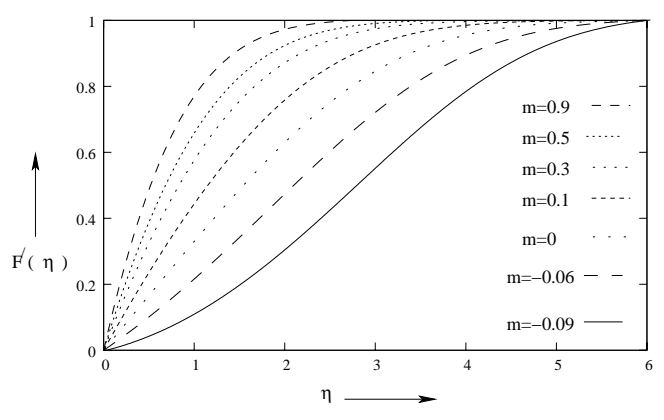

Fig. 3(a) : Variation of velocity $F^{\prime}(\eta)$ with $\eta$ for several values of $m$ with $\mathrm{a}=1, \mathrm{~A}=0, \mathrm{Q}=2$ and $\mathrm{Pr}=0.5$.

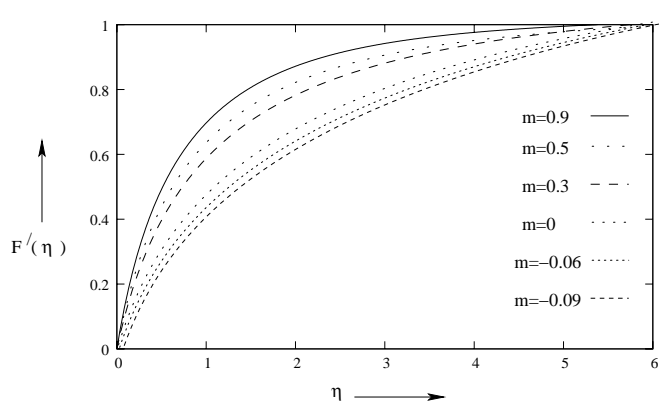

Fig. 3(b) : Variation of velocity $F^{\prime}(\eta)$ with $\eta$ for several values of $m$ with $a=1, A=10, Q=2$ and $\operatorname{Pr}=0.5$.

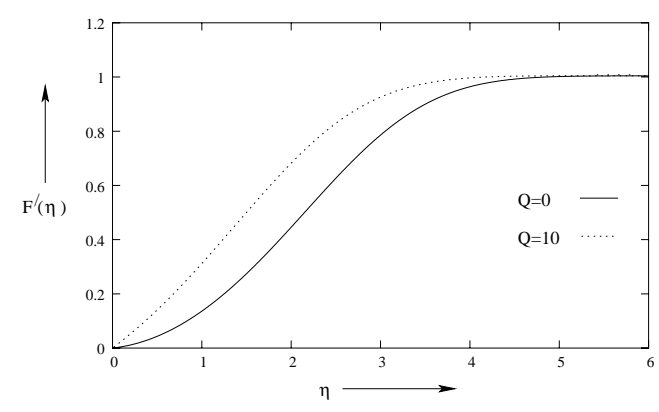

Fig. 4: Variation of velocity $F^{\prime}(\eta)$ with $\eta$ for $m=-0.091$ for two two values $\mathrm{Q}$ with $\mathrm{a}=1, \mathrm{~A}=0$ and $\mathrm{Pr}=0.5$.

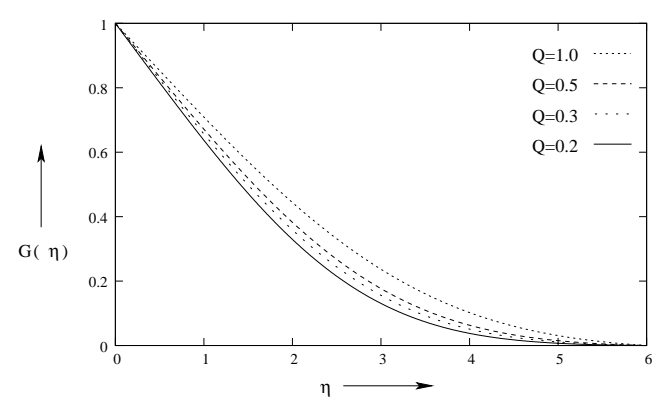

Fig. 5 : Variation of temperature $\quad$ G $(\eta)$ with $\eta$ for several values of $\mathrm{Q}$ with $\mathrm{a}=1, \mathrm{~A}=10, \mathrm{~m}=0.5$ and $\mathrm{Pr}=0.5$.

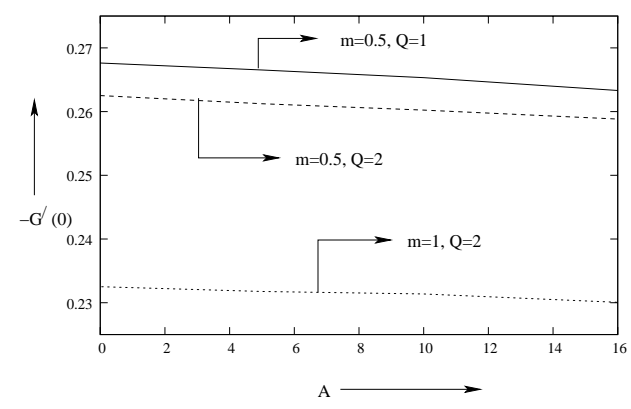

Fig. 6: Cariation of rate of heat transfer $\left[-\mathrm{G}^{\prime}(0)\right]$ with $\mathrm{A}$ for two different values of $\mathrm{m}$ and $\mathrm{Q}$ with $\mathrm{a}=1, \mathrm{~A}=0$ and $\mathrm{Pr}=0.5$. 